\section{Good touch, bad touch}

\section{By Lev Osherovich, Senior Writer}

University of California, San Francisco researchers have identified the precise spinal cord neurons that cause hypersensitivity to gentle touches, a common form of neuropathic pain associated with injury and inflammation. ${ }^{1}$ The study also presents a drug target specific to these neurons: a glutamate transporter called VGLUT3 that helps prime neurons to fire at the lightest touch.

Physiologists have known since the 1920s that different neurons within the spinal cord's dorsal root ganglia convey distinct types of pain to the brain. These neurons include fast-acting, highly myelinated nerve fibers that convey acute pain caused by heat or injury, and slow-acting, poorly myelinated fibers that transmit more deep-rooted, throbbing pain caused by inflammation.

Harmless touching such as gentle brushing of the skin, as well as painful contact with injured tissue, are sensed relatively quickly. As a result, researchers historically believed the transmission of these signals came from fastacting fibers.

"Previously, the idea in the field had been that the primary fibers responsible for light mechanical touch sensation were large myelinated fibers," said Michael Salter, a professor of physiology at the University of Toronto who was not involved in the study. "It wasn't really

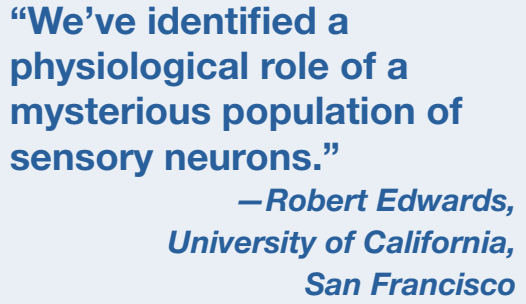

suggesting that they formed slow-acting fibers.

The team then examined pain sensation in VGLUT3 knockout mice. The animals had normal aversion to heat and cold but had a slightly delayed response to tail-pinching, a test of their sensitivity to acute mechanical pressure. Notably, knockouts with chemically induced injuries to their paws exhibited a greater pain threshold and a slower response to light touching of the injured paw than wild-type controls.

Altogether, the VGLUT3 knockouts had a selective defect in their response to mechanical pain, which is heightened during injury and inflammation.

"Their acute sensation was relatively normal," said Edwards. "But in models of chronic pain due to inflammation and nerve injury, these mice don't develop the same sort of mechanical hypersensitivity" as wild-type mice.

\section{Selective disruption}

Now that they have identified the neurons involved in mechanical pain, the UCSF team's next goal is to selectively inactivate the neurons to treat neuropathic pain. According to Edwards and Salter, VGLUT3 has the makings of an ideal target.

"VGLUT3 is responsible for pumping glutamate into synaptic vesicles," an essential early step for neurotransmission, said Salter. "If you inhibited this target, you might inhibit the ability of these neurons to respond to touch."

Also, the protein is located in the peripheral nervous system and should be more druggable than targets in the CNS, which is protected by the blood brain barrier.

According to Salter, the challenge is to selectively inhibit the VGLUT3-expressing neurons without disrupting the activity of other types of glutamatergic neurons in other parts of the nervous system. Because glutamate is the main excitatory neurotransmitter, blocking its signalclear what the role of slow-acting fibers was."

Now, the UCSF team thinks it has overturned the previous theory by pinpointing a small population of slow-acting fibers that are the main messengers of mechanical hypersensitivity.

"We've identified a physiological role of a mysterious population of sensory neurons," said Robert Edwards, professor of neurology and physiology at UCSF and lead author of the paper in Nature that described the findings. "People have known about these neurons for a long time but didn't know what they did."

Edwards' team discovered the role of the slow-acting fibers while characterizing the expression pattern of the intracellular glutamate transporter VGLUT3 (solute carrier family 17, sodium-dependent inorganic phosphate cotransporter, member 8; SLC17A8). The team had previously identified VGLUT3 as a transporter needed for glutamatergic neuron activity.

The group found that a small subset of neurons in the dorsal root ganglia expressed VGLUT3. Those cells also had low levels of myelin, ing systemically would have many undesired side effects, he said.

Although all glutamatergic neurons require a transporter like VGLUT3 to load up synaptic vesicles, VGLUT3 appears to function primarily in the pain-sensing neurons. Edwards said neurons in other parts of the nervous system rely on other transporters. Thus, selectively inhibiting VGLUT3 might avoid the harmful effects of broadly inhibiting glutamate transport or signaling.

At the same time, antagonizing VGLUT3 could potentially synergize with direct inhibition of glutamate receptors. However, Bruno Oesch, CEO of Neurotune AG, said it is unknown whether the neurons downstream of VGLUT3-containing cells use NMDARs or other glutamate receptors such as AMPARs and metabotropic glutamate receptors to transmit pain signals to the brain.

At least 14 companies have glutamate-modulating compounds in development for various pain indications. The majority antagonize NMDA-type glutamate receptors. Neurotune's NT-11624 dimirace- 


\section{TARGETS \& MECHANISMS}

tam, a small molecule modulator of NMDA-type glutamate receptors, is expected to complete Phase II trials for HIV-associated neuropathic pain in mid-2010.

Oesch said NT-11624 is selective for spinal cord neurons and thus avoids concerns about systemic glutamate inhibition.

There are some worries about the side effects of blocking VGLUT3. Edwards noted that the VGLUT3 knockout mice were completely deaf and prone to seizures. And a study published earlier this year suggests that the same neurons identified by Edwards' team are also needed for pleasant touch sensation. ${ }^{2}$

"Targeting the new transporter protein may be interesting but may also be very risky," said Oesch. The developmental and sensory defects in VGLUT3 knockout mice could mean that "manipulating VGLUT3 may cause derangements in movement and cognition," he added.

Salter suggested that the side effects in the VGLUT3 knockout phenotype might not be as severe if the transporter were only partially inhibited.
Edwards' next steps are to characterize the signals upstream of the VGLUT3-expressing neurons and to identify the downstream glutamate receptors of these neurons.

$\mathrm{He}$ is also developing a cell culture assay for VGLUT3 activity and is planning to screen for selective inhibitors of the transporter. Edwards has filed patents on targeting VGLUT3 to treat pain and is seeking industry partners to aid with small molecule screening.

Osherovich, L. SciBX 2(46); doi:10.1038/scibx.2009.1685

Published online Dec. 3, 2009

REFERENCES

1. Seal, R.P. et al. Nature; published online Nov.15, 2009; doi:10.1038/nature08505

Contact: Robert Edwards, University of California, San Francisco, Calif. e-mail: Robert.Edwards@ucsf.edu

2. Löken, L.S. et al. Nat. Neurosci. 12, 547-548 (2009)

COMPANIES AND INSTITUTIONS MENTIONED

Neurotune AG, Schlieren-Zurich, Switzerland

University of California, San Francisco, Calif.

University of Toronto, Toronto, Ontario, Canada 